\title{
MANOBRAS De SWING-BY PROPUlSAdA FORA dO PERIAPSIDE
}

\author{
Alessandra F. DA SILVA ${ }^{1}$, ANTÔNIO F. B. A. PRADO ${ }^{1}$, OTHON C. WINTER ${ }^{2}$.
}

\author{
1. Instituto Nacional de Pesquisas Espaciais- INPE \\ 12227-010, São José dos Campos, SP, Brasil \\ E-mails: aleferrazsilva@hotmail.com, prado@dem.inpe.br \\ 2. Grupo de Dinâmica Orbital e Planetologia, Univ. Estadual Paulista-UNESP \\ 12516-410, Guaratinguetá, SP, Brasil \\ E-mails: ocwinteregmail.com
}

\begin{abstract}
Resumo — Existe vários estudos na literatura sobre Manobras de Swing-By (também conhecidas como Manobras de Flyby). Este tipo de manobra é importante, pois gera uma economia de combustível que torna viável um grande número de missões que seriam impossíveis de serem realizadas sem esse conceito. Essa manobra pode ser estudada de forma independente ou combinada com a aplicação de forças propulsivas. Neste trabalho foi desenvolvido um algoritmo para o cálculo da variação da energia de uma manobra combinada onde a aplicação do impulso ocorre antes ou depois do periapside da órbita do veículo espacial, na vizinhança da esfera de influência do corpo secundário. A partir deste algoritmo inferiu-se que o ponto ótimo para aplicar o impulso é, em geral, fora do periapside.
\end{abstract}

Palavras-chave — Swing-By, manobras impulsivas, trajetória de satélite, periapside

\section{Introdução}

A manobra de Swing-By (também chamada de manobra de Flyby) consiste em uma passagem de um veículo espacial próximo a um corpo celeste com o objetivo de alterar a sua órbita. Essa alteração é feita com o uso da gravidade do corpo celeste próximo, e inclui modificação da velocidade, energia e momento angular do veículo espacial. O objetivo maior desta manobra é a economia de combustível em missões espaciais, já que o Swing-By é equivalente à aplicação de um impulso com gasto de combustível zero.

A manobra de Swing-By propulsado é a combinação do Swing-By padrão com um impulso aplicado no veículo espacial durante o encontro próximo. $\mathrm{O}$ propulsor usado para a aplicação do impulso depende da missão.

A literatura mostra no trabalho de Prado (1996), como funciona a manobra de Swing-By propulsado com a condição de que o impulso seja aplicado no veículo espacial exatamente no instante de sua passagem pelo periapside da órbita (ponto mais próximo entre o veículo espacial e o corpo secundário) e em um ponto após a saída do veículo espacial da esfera de influência do corpo celeste. Isso é feito para estudar a eficiência dessa manobra combinada, ou seja, deseja-se saber em que circunstância é mais interessante aplicar o impulso, durante a passagem próxima ou após essa passagem. Há outros trabalhos sobre o assunto disponíveis na literatura como: Dunham e Davis (1985), Striepe e Braun (1991), Prado (1993), Prado e Broucke (1995), Petropoulos e Logunski (2000), McConaghy et. al. (2003), Araújo et. al. (2008), Gomes e Prado (2009), Sukhanov et. al. (2010) e outros.

Neste trabalho foi desenvolvido um algoritmo para o cálculo da variação da energia e do momento angular de uma manobra combinada onde a aplicação do impulso ocorre antes ou depois do periapside da trajetória de passagem próxima, mas na vizinhança da esfera de influência do corpo secundário. Segundo Araújo (2007), esfera de influência é uma região definida por um raio que é uma espécie de fronteira a partir da qual uma partícula com uma dada velocidade relativa está sendo significativamente influenciada pelo corpo secundário.

\section{Dinâmica do Sistema}

No presente trabalho utilizamos o problema restrito de três corpos e a regularização de Lemaître, com o objetivo de evitar singularidades na integração numérica.

O sistema é formado por três corpos: $\mathrm{M}_{1}$, o corpo primário (corpo com maior massa), $\mathrm{M}_{2}$, o corpo secundário que executa uma órbita kepleriana em torno de $\mathrm{M}_{1}$, e $\mathrm{M}_{3}$, um satélite com uma massa que pode ser assumida desprezível, que orbita $M_{1}$ e faz uma manobra de Swing-By com $\mathrm{M}_{2}$.

Neste trabalho usamos como referência o sistema rotacional, ou seja, $M_{1}$ e $M_{2}$ ficam fixos neste sistema.

As equações do movimento do problema restrito de três corpos são (Murray e Dermott, 1999):

$$
\begin{gathered}
\ddot{x}-\dot{2} \dot{y}=\frac{\partial \Omega}{\partial x} \\
\ddot{y}+2 \dot{x}=\frac{\partial \Omega}{\partial y} \\
\Omega=\frac{1}{2}\left(x^{2}+y^{2}\right)+\frac{1-\mu}{r_{1}}+\frac{\mu}{r_{2}}
\end{gathered}
$$


A equação do movimento depende de $\Omega$, e $\Omega$ depende de $r_{1}$ e $r_{2}$ no denominador. Quando $M_{3}$ se aproxima de um dos primários, os valores de $r_{1}$ (distância entre $M_{1}$ e $M_{3}$ dividida pela distância $M_{1}-M_{2}$ ) e $r_{2}$ (distância entre $M_{2}$ e $M_{3}$ dividida pela distância $\mathrm{M}_{1}-\mathrm{M}_{2}$ ) ficam muito pequenos, ficando próximo de uma singularidade.

O objetivo da técnica de regularização é eliminar essas singularidades por meio da substituição de variáveis (Lapa, 2008).

\section{Método Proposto}

O objetivo é estudar o efeito da aplicação do impulso antes e depois do Swing-By, fora do periapside da órbita, mas na vizinhança da esfera de influência de $\mathrm{M}_{2}$.

Foi usado um raio igual à metade da distância entre $M_{1}$ e $M_{2}$ para calcular a energia antes e depois da passagem do satélite pelo periapside. Essa distância é grande o suficiente para garantir, que fora dela, o corpo secundário não influência mais na trajetória do satélite.

A partir dos resultados obtidos foi possível verificar o ponto de maior eficiência para se aplicar o impulso em uma manobra. Com isso, foi possível complementar o estudo efetuado por Prado (1996) e mapear regiões ótimas para a aplicação do impulso entre as opções: a) no instante da passagem pelo periapside; b) fora do periapside, mas dentro da esfera de influência de $\mathrm{M}_{2}$; c) fora da esfera de influência de $\mathrm{M}_{2}$.

A Figura 1 descreve em detalhes esse novo tipo de manobra considerada. A posição $\mathbf{r}=(\mathrm{x}, \mathrm{y})$ do ponto onde foi aplicado o impulso $\delta \mathrm{V}$ foi especificada por meio da anomalia verdadeira, denominada $\theta$, desse ponto. Foi adotado para o deslocamento de $\theta$ o sentido anti-horário como positivo e o sentido horário como negativo, ambos medidos a partir do vetor $\mathbf{r}_{\mathrm{p}}=\left(\mathrm{x}_{\mathrm{p}}, \mathrm{y}_{\mathrm{p}}\right)$. A curva preta representa a primeira órbita e a curva cinza representa a segunda órbita.

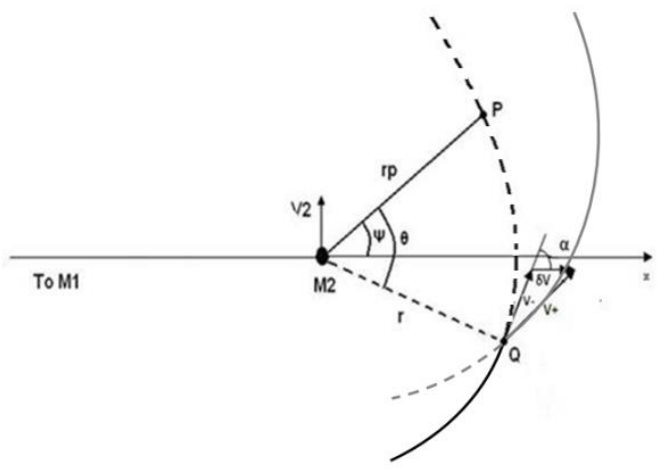

Figura 1 - Geometria do Swing-By com aplicação do impulso fora do periapside

Conforme apresentado na Figura 1, a posição onde foi aplicado o impulso é uma função de $\theta$. Esse ângulo $\theta$ pode ser obtido a partir do produto escalar dos vetores $\mathbf{r}_{\mathbf{p}}$ e $\mathbf{r}$. Utilizando esse enfoque, chegamos na Equação 4.

$$
\theta=\arccos \left(\frac{\mathrm{x}_{\mathrm{p}} \mathrm{X}+\mathrm{Y}_{\mathrm{p}} \mathrm{Y}}{r_{\mathrm{p}} r}\right)
$$

Sendo assim, o algoritmo para solução numérica do problema foi feito da seguinte forma:

a) Iniciou-se o estudo com o veículo espacial colocado no ponto P. Isso foi feito especificando-se as três variáveis que definem univocamente uma trajetória de Swing-By: $V_{p}$, magnitude da velocidade do satélite ao passar pelo periapside; $r_{p}$, menor distância entre o veículo espacial e o corpo celeste, chamado raio do periapside; e $\psi$, ângulo entre a linha que liga os dois primários até a linha do periapside;

b) A partir desse ponto $P$ efetuou-se uma integração numérica, reversa ou direta de acordo com o sinal de $\theta$, até que o veículo espacial atingisse o ponto $\mathrm{Q}$, mostrado na Figura 1. Esse ponto foi definido através da especificação do valor do ângulo $\theta$;

c) A partir do ponto $Q$ executou-se uma integração reversa no tempo (Vieira Neto \& Winter, 2001), até que se atingisse a metade da distância entre $\mathrm{M}_{1}$ e $\mathrm{M}_{2}$, para obter a primeira órbita e os valores de energia, velocidade e momento angular antes da manobra completa;

d) Aplicou-se um impulso $\delta \mathrm{V}$ no ponto $\mathrm{Q}$, formando um angulo $\alpha$ entre a direção do impulso e a direção do movimento do veiculo espacial, para obter a nova órbita;

e) Integrou-se para frente no tempo essa nova órbita até um ponto distante de $\mathrm{M}_{2}$, obtendo o valor da energia da manobra completa;

f) Por fim, foi calculada a variação da energia em função da magnitude do impulso, do ângulo de aplicação do impulso e do ângulo $\theta$, que define o local de aplicação do impulso. Essa grandeza pode então ser escrita como:

$$
\Delta E=f(\delta V, \alpha, \theta)
$$

A variação da energia foi calculada pela diferença entre a energia da órbita obtida depois da aplicação do impulso e da órbita que se tinha anteriormente.

\subsection{Resultados}

Foi considerado $\alpha=0^{\circ}$, ou seja, o vetor impulso está sempre na mesma direção do vetor velocidade do satélite (na primeira órbita), incrementando a energia.

A anomalia verdadeira $(\theta)$ é o ângulo que define o ponto onde será aplicado o impulso na manobra. Quando $\theta \neq 0^{\circ}$, significa que o impulso será aplicado fora do periapside da órbita.

Serão apresentados os resultados para o sistema Terra-Lua-satélite, que resultaram em variação máxima de energia, com raio do periapside igual a 1.1 
raios da Lua e diferentes valores da magnitude do impulso.

Estudamos somente os casos com aplicação do impulso na direção tangencial da órbita $\left(\alpha=0^{\circ}\right)$. As Figuras 2 e 3 mostram que os melhores pontos de aplicação do impulso estão fora do periapside da órbita. Quando $\psi=0^{\circ}$, a magnitude do impulso aumenta e $\theta$ tende a zero. Isso ocorre porque, como o impulso domina a manobra, ele tende a alinhar impulso com velocidade.

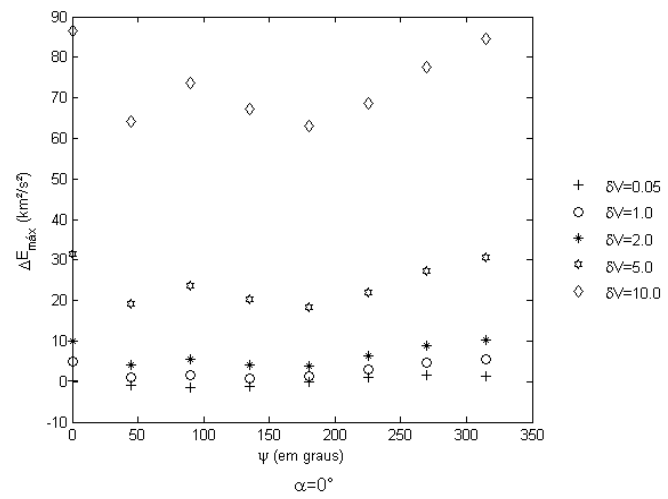

Figura 2 - Gráfico de $\psi \mathrm{Vs} \Delta \mathrm{E}_{\max }$, para $\alpha=0^{\circ}$ e diferentes valores de impulso.

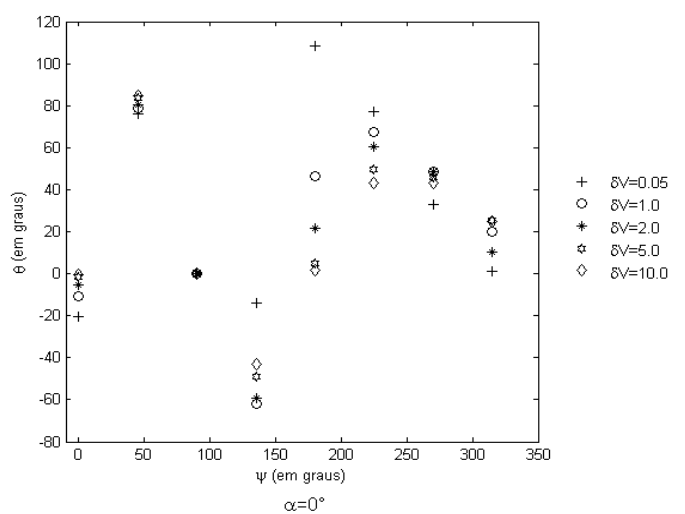

Figura 3 - Gráfico de $\psi$ Vs $\theta$, para $\alpha=0^{\circ}$ e diferentes valores de impulso

Temos que se o satélite passa na frente do corpo secundário $\left(0^{\circ}<\psi<180^{\circ}\right)$, ele é freado pelo corpo e perde energia. A perda máxima ocorre quando $\psi=$ $90^{\circ}$. Se o satélite passa atrás do corpo secundário $\left(180^{\circ}<\psi<360^{\circ}\right)$, ele é acelerado pelo corpo e ganha energia. $\mathrm{O}$ ganho máximo de energia ocorre quando $\psi=270^{\circ}$ (Prado, 2001).

Neste trabalho o objetivo é apresentar as condições que resultam na variação máxima de energia, quando o impulso é aplicado fora do periapside da órbita e na direção do satélite. Logo sabemos que os melhores resultados tendem a estar entre $180^{\circ}<\psi<$ $360^{\circ}$. É importante ressaltar que essas são regiões de ganho e perda de energia baseados no periapside antes do impulso. Porém, como o periapside da órbita é alterado com a aplicação do impulso, mesmo essas regras podem ser alteradas. $\mathrm{O}$ periapside da órbita original ainda tem efeito sobre a trajetória, já que ele define o ponto de aplicação do impulso e, em geral, o novo periapside tende a não estar tão distante do periapside da órbita original. Esse comportamento é válido para todos os casos em que $\theta$ varia.

Será apresentada a variação da energia de alguns casos que estão nessa região. Ver Figuras de 4 a 9.

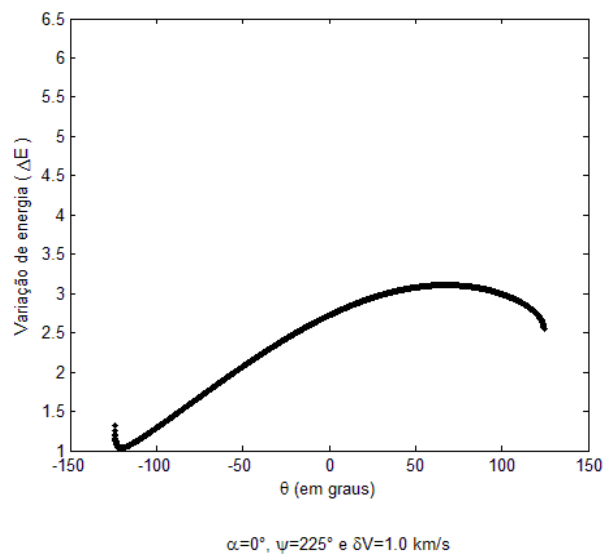

Figura 4 - Comportamento da variação da energia em função da anomalia verdadeira para $\alpha=0^{\circ}$.

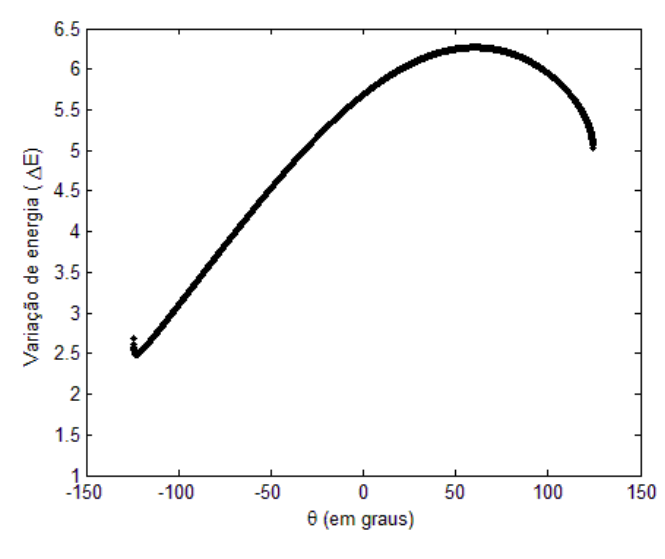

$\alpha=0^{\circ}, \psi=225^{\circ}$ e $\delta \mathrm{V}=2.0 \mathrm{~km} / \mathrm{s}$

Figura 5 - Comportamento da variação da energia em função da anomalia verdadeira para $\alpha=0^{\circ}$

O ponto de aplicação do impulso é calculado deslocando o valor de $\theta$ (no sentido horário ou antihorário, dependendo do sinal) a partir da linha do periapside $r_{p}$. No caso da Figura 4 e 5 o valor de $\theta$, para a variação máxima de energia é aproximadamente $67^{\circ}$ e $60^{\circ}$, respectivamente. Isso significa que o impulso foi aplicado na região em que $180^{\circ}<\psi<$ $360^{\circ}$. Região essa em que se ganha energia devido ao Swing-By padrão. 


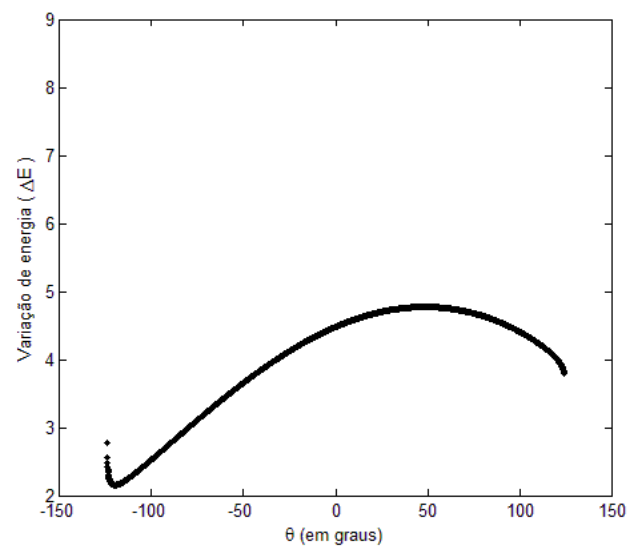

$\alpha=0^{\circ}, \psi=270^{\circ}$ e $\delta V=1.0 \mathrm{~km} / \mathrm{s}$

Figura 6 - Comportamento da variação da energia em função da anomalia verdadeira para $\alpha=0^{\circ}$.

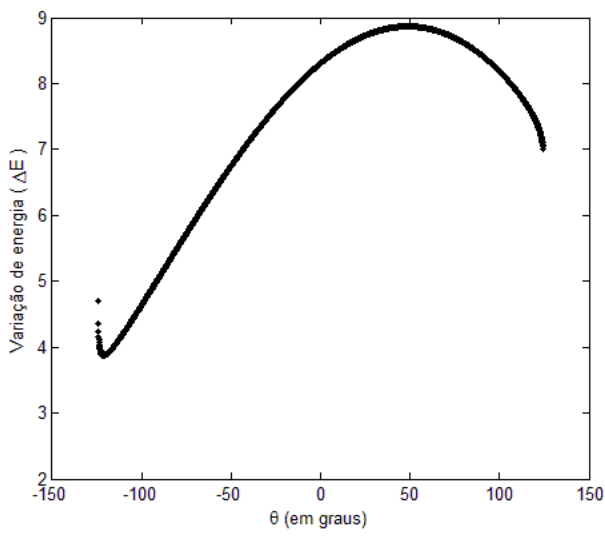

$\alpha=0^{\circ}, \psi=270^{\circ}$ e $\delta V=2.0 \mathrm{~km} / \mathrm{s}$

Figura 7 - Comportamento da variação da energia em função da anomalia verdadeira para $\alpha=0^{\circ}$.

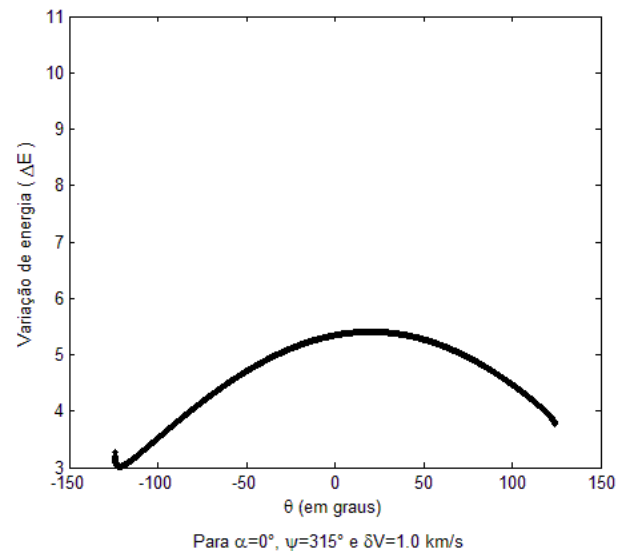

Figura 8 - Comportamento da variação da energia em função da anomalia verdadeira para $\alpha=0^{\circ}$.

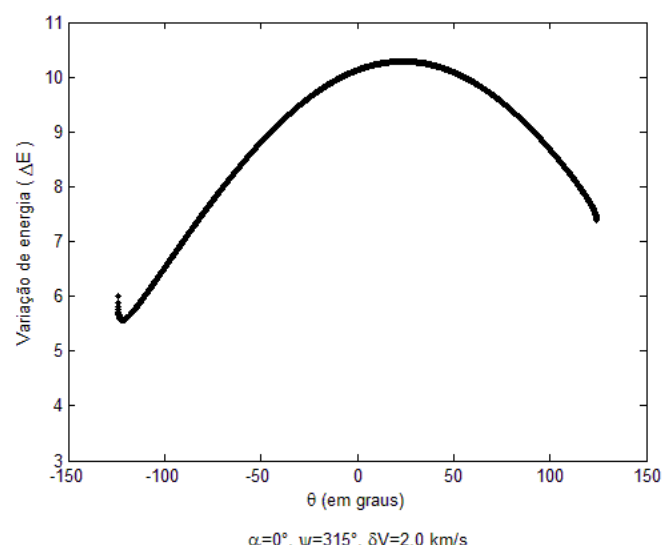

Figura 9 - Comportamento da variação da energia em função da anomalia verdadeira para $\alpha=0^{\circ}$.

Também para as Figuras 6, 7, 8 e 9 o impulso foi aplicado na região que se ganha energia devido ao Swing-By padrão, colaborando na obtenção da variação máxima de energia.

Todos os casos com $\alpha=0^{\circ}$ foram simulados $\operatorname{com} \theta$ entre $-180^{\circ}$ e $180^{\circ}$. Nos gráficos onde há descontinuidade das figuras ou as curvas não alcançam valores de $-180^{\circ}$ ou $180^{\circ}$ (em $\theta$ ) significa que ocorreram condições de capturas do satélite pela Lua.

\section{Conclusão}

Foram feitas simulações estudando o efeito de $\theta$ apenas, adotando $\alpha=0^{\circ}$ mostrando que mesmo com a aplicação do impulso na direção tangencial a órbita, os melhores resultados estão fora do periapside.

A princípio pode parecer óbvio que o melhor local para aplicação do impulso seja no ponto $(0,0)$, ou seja, na direção de movimento do satélite e no periapside da órbita. Porém não é isso que acontece, porque quando se aplica um impulso em condições diferentes destas, pode-se aumentar ou diminuir a variação de energia devido ao Swing-By, com isso compensando o menor ganho de energia devido ao impulso. Isso ocorre porque a órbita muda instantaneamente, logo seus parâmetros também mudam, incluindo um novo $r_{p}$, que poderá ser menor (essa mudança irá depender das condições iniciais, pode-se querer maximizar o efeito do Swing-By e então fazer com que $r_{p}$ seja maior) e consequentemente ter um Swing-By mais eficiente. O resultado líquido desta troca é positivo. Por isso a conclusão de que aplicação do impulso fora do periapside é a mais eficiente, além também de ser flexível.

\section{Agradecimentos}

Os autores desejam expressar seu agradecimento pelo apoio prestado por doações \# 473387/2012-3 e 304700/2009-6, do Conselho Nacional de Desenvolvimento Científico e Tecnológico (CNPq); subsídios \# 2011/09310-7 e 2011/08171 -3, da Fundação de 
Pesquisa de São Paulo (FAPESP) e com o apoio financeiro do Conselho Nacional de Aperfeiçoamento de Ensino Superior (CAPES).

\section{Referências Bibliográficas}

Araújo, R. A. Encontros próximos: captura gravitacional temporária e esfera de influência. 2007. f. 112. Dissertação (Mestrado em Física) - Universidade Estadual Paulista - Faculdade de Engenharia de Guaratinguetá. Guaratinguetá, 2007.

Araújo R. A. N.; Winter O. C.; Prado A. F. B. A.; Vieira Martins, R. 2008. Sphere of influence and gravitational capture radius: a dynamical approach. Monthly Notes of the Royal Astronomical Society, v. 391 675. DOI: 10.1111/ j.1365-2966.2008.13833.x

Dunham, D.; Davis, S. Optimization of a Multiple Lunar- Swing-By Trajectory Sequence. Journal of Astronautical Sciences, v. 33, n. 3, p. 275288, 1985.

Gomes, V.M.; Prado, A.F.B.A. A Study of the Close Approach Between a Planet and a Cloud of Particles. Recent Advances in Signal Processing, Robotics and Automation, p. 126-131. ISBN 978-960-474-054-3, Cambridge, England, 2009.

Lapa, L.C. Regularização de singularidades em mecânica celeste. 2008. f. 80. Dissertação (Mestrado em Matemática) - Universidade Federal de Pernambuco, Recife, 2008.

McConaghy, T. T.; Debban, T. J.; Petropoulos, A. E.; Logunski, J. M. Design and optimization of lowthrust gravity trajectories with gravity assist. Journal of Spacecraft and Rockets, v. 40, n. 3, p. 380-387, 2003. DOI: $10.2514 / 2.3973$
Murray C. D., Dermott S. F., 1999, Solar System Dynamics, 1 edn. Cambridge University Press.

Petropoulos, A. E.; Longuski, J. M. Automated design of low-thrust gravity-assist trajectories," AIAA Paper 2000-4033, 2000.

Prado, A. F. B. A. Optimal transfer and swing-orbits in the two-and three-body problems. 1993. 253 p. (INPE-5572-TAE/022). Tese de Doutorado Faculty of the Graduate School. University of Texas, Austin, 1993

Prado, A. F. B. A. Powered swing-by. Journal of Guidance Control and Dynamics, v. 19, n. 5, p. 1142-1147, Sept. - Oct. 1996. (INPE-6732PRE/2754). DOI: $10.2514 / 2.3973$

Prado, A. F. B. A. Trajetórias espaciais e manobras assistidas por gravidade. São José dos Campos: INPE, 2001. 171 p. ISBN 85-17-00003-X.

Prado, A.F.B.A ; Broucke, R. A. A Classification of Swing-By Trajectories Using the Moon. Applied Mechanics Reviews, v. 48, n.11, p. 138-142, 1995. DOI: $10.1115 / 1.3005063$

Striepe, S.A.; Braun, R.D. Effects of a Venus Swing by Periapsis Burn During an Earth-Mars Trajectory. Journal of the Astronautical Sciences, 39(3), 299-312, 1991.

Sukhanov, A. Velho, H. F. C.; Macau, E. ; Winter, O. The Aster Project: Flight to a Near-Earth Asteroid 443-450, 2010.

Vieira Neto, E. \& Winter, O. C., Time analysis for temporary gravitational capture: satellites of Uranus. Astronomical Journal, v. 122, p. 440, 2001. DOI: $10.1086 / 321101$ 